\title{
A Hard X-Ray Study of a
}

Manganese-Terpyridine Dimer

Catalyst in a Chromium-Based Metal

Organic Framework

\section{Alexandra Ramsey}

Office of Science, Science Undergraduate Laboratóry Internship (SULI) Program



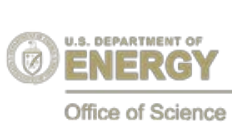

This work was supported in part by the U.S. Department of Energy, Office of Science, Office of Workforce Development for Teachers and Scientists (WDTS) under the Science Undergraduate Laboratory Internship (SULI) program, under Contract No. DE-AC02-76SF00515. 


\section{ABSTRACT}

Cleaner forms of energy are needed, and $\mathrm{H}_{2}$ produced from water splitting is a possible source. However, a robust catalyst is necessary to carry out the water oxidation reaction. Plants utilize Photosystem II to catalyze water oxidation as a part of photosynthesis, and many synthetic water oxidation catalysts use Photosystem II as a model. In this study, the catalyst of interest was [(terpy) Mn( $\mu$-O)2Mn(terpy)]3+ (MnTD), which was synthesized in a chromium-based Metal Organic Framework (MOF) to avoid degradation of MnTD molecules. Hard X-ray powder diffraction was the primary method of analysis. The diffraction data was used to detect the presence of MOF in samples at different catalytic stages, and lattice parameters were assigned to the samples containing MOF. Fourier maps were constructed to determine the contents of the MOF as preliminary studies suggested that MnTD may not be present. Results showed that MOF is present before catalysis occurs, but disappears in the initial stages of catalysis. Changes in the MOF's lattice parameters suggest attractive interactions between the MOF and catalyst; these interactions may lead to the observed MOF degradation. Fourier maps also reveal limited, if any, amounts of MnTD in the system. Molecular manganese oxide may be the source of the high rate of water oxidation catalysis in the studied system. 


\section{Water Splitting}

$2 \mathrm{H}_{2} \mathrm{O} \rightarrow \mathrm{O}_{2}+4 \mathrm{H}^{+}+4 \mathrm{e}^{-} \quad 4 \mathrm{H}^{+}+4 \mathrm{e}^{-} \rightarrow 2 \mathrm{H}_{2}$
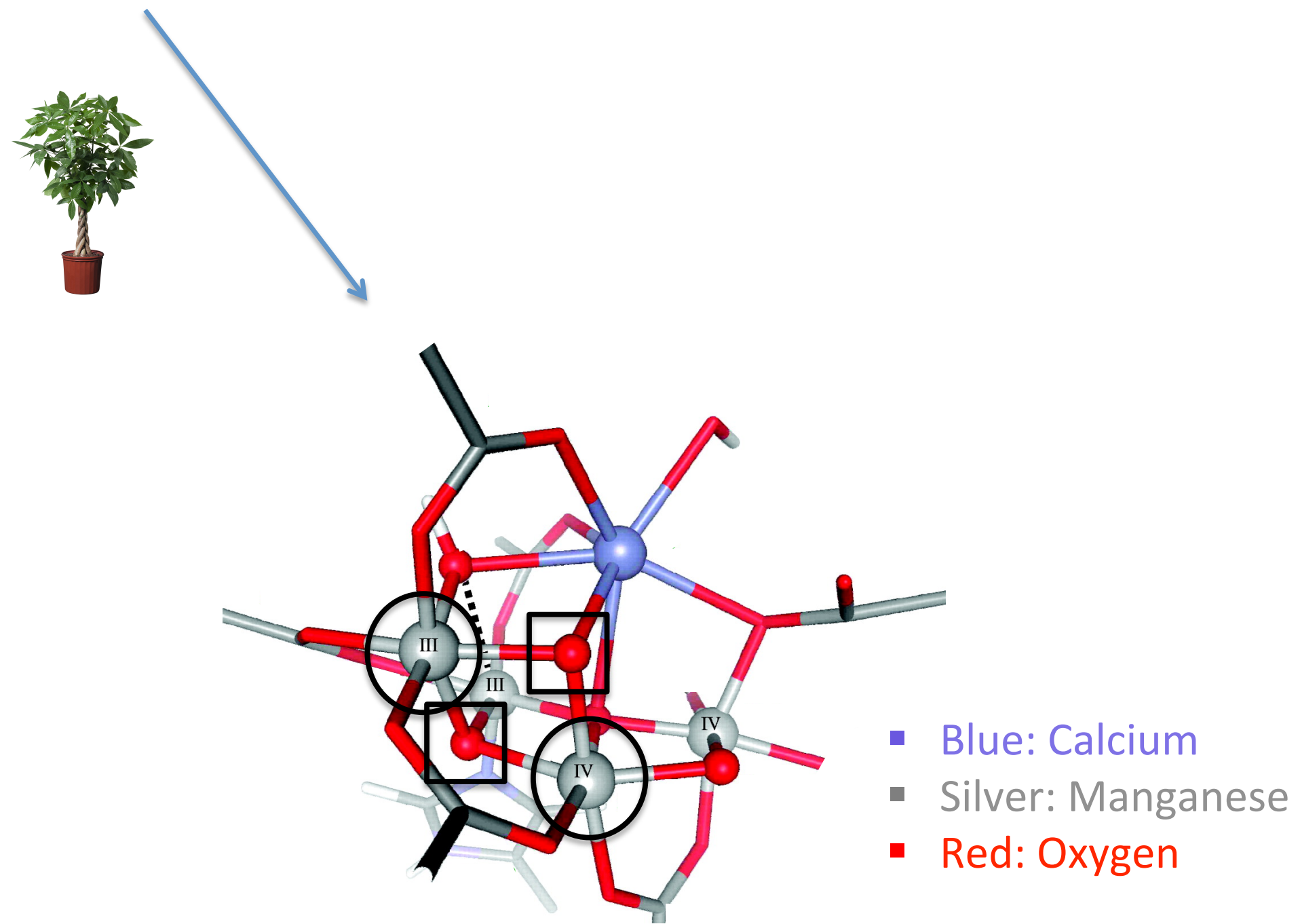

Dau, H., et al., The Royal Society 363 (1494), 1237-1244 (2008). 


\section{The Manganese-Terpyridine (terpy) Dimer}

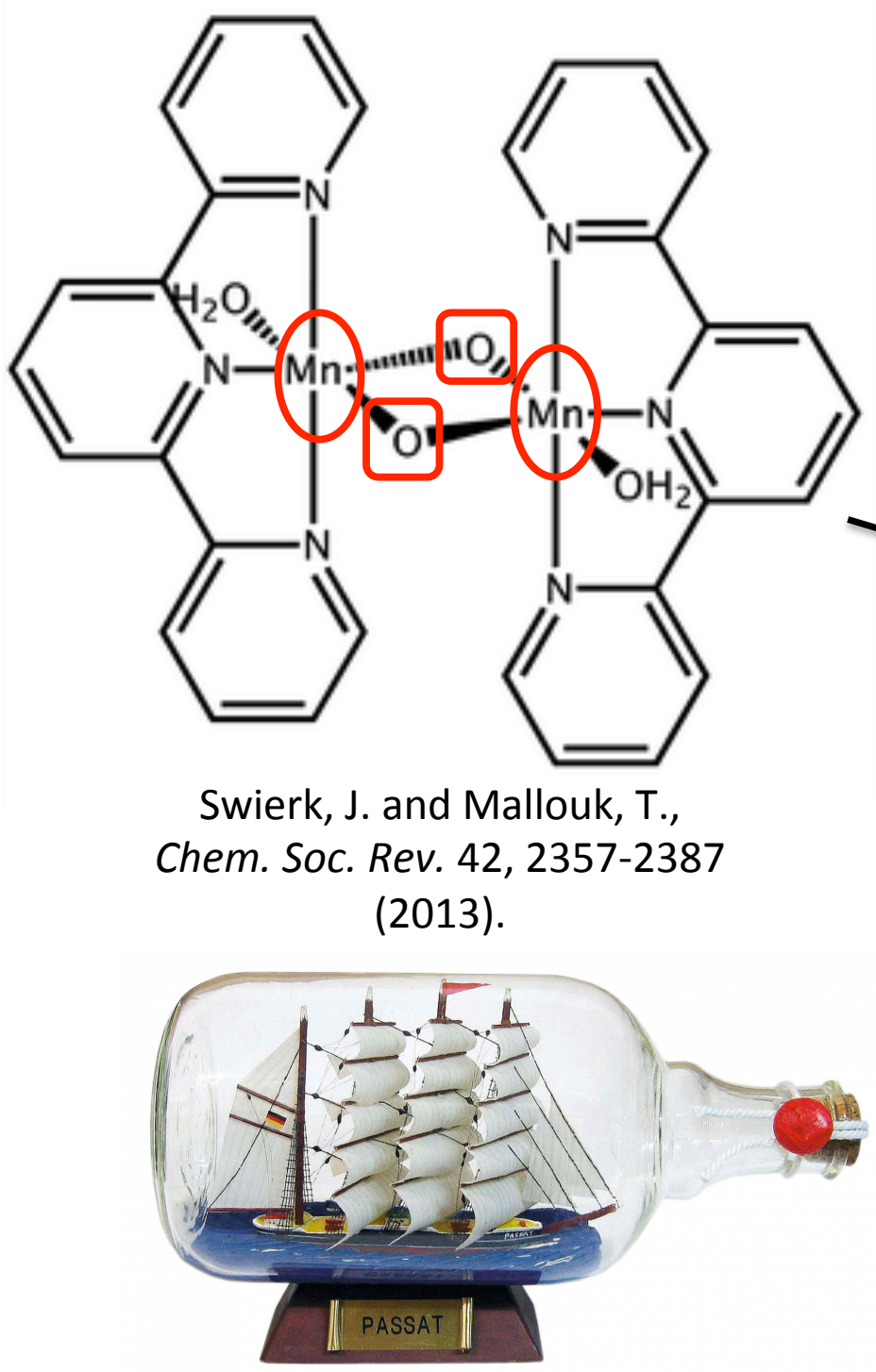

Metal Organic Framework (MOF)

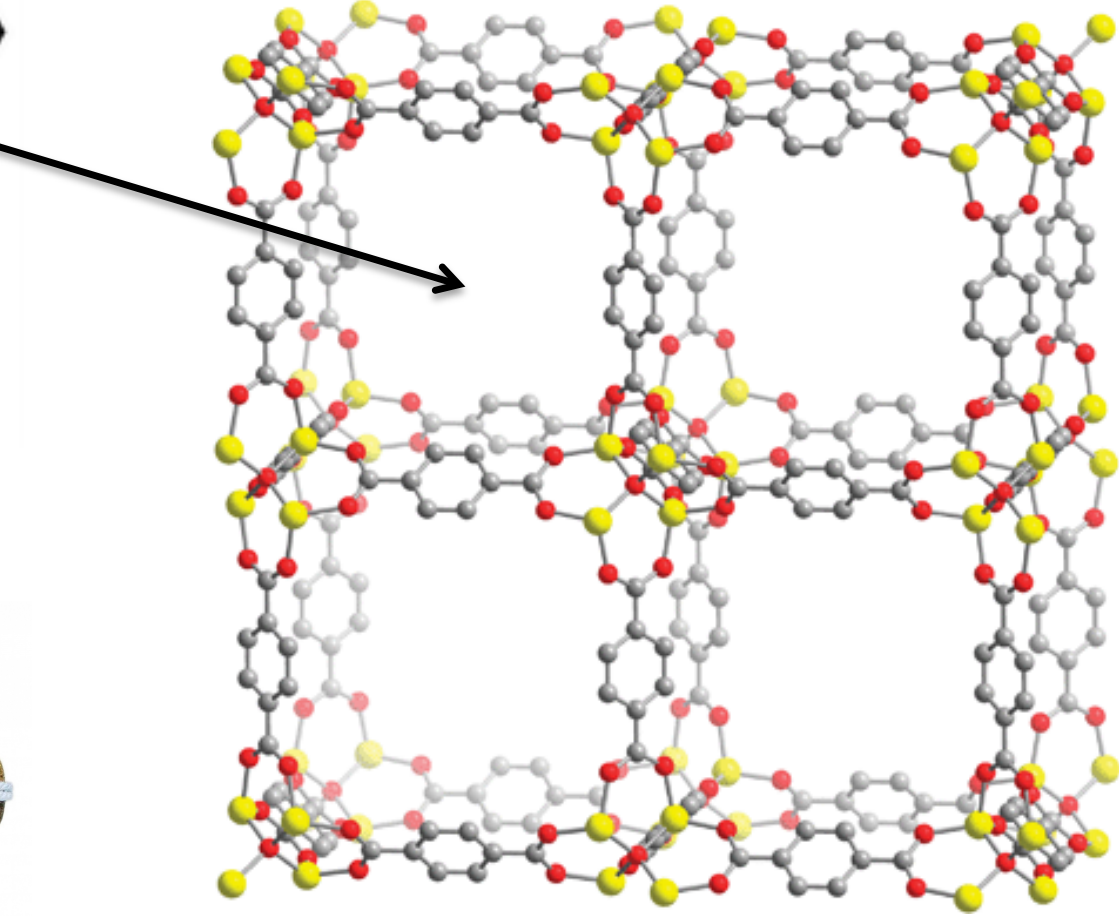

Murray, L., Dinca, M., and Long, J., Chem. Soc. Rev. 38, 1294-1314 (2009).

http://www.mare2.com/

popup_image.php/pID/655/imgID/0 


\section{$\underline{\text { Questions }}$}

- Is the manganese-terpy dimer the catalytically active molecule?

- Has the manganese-terpy dimer been synthesized inside of the MOF?

O MOF

- MOF in acetate buffer

- MOF with catalyst but before catalysis occurs

- MOF with activated catalyst after 45 minutes of catalysis

- MOF with activated catalyst after 2 hours of catalysis 


\section{SSRL}



4.

4xisen

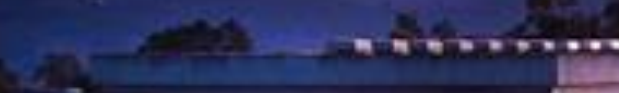

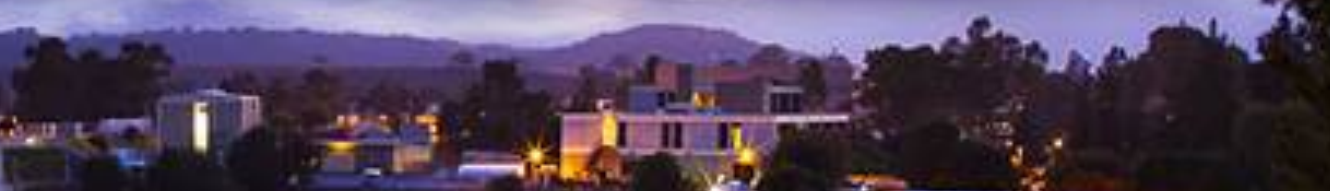
8.

$x^{2} \rightarrow x^{2}+x^{2}=0$



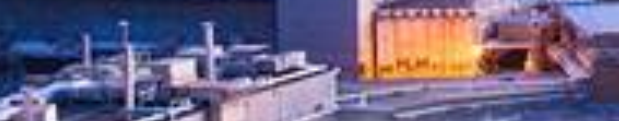

at 11 . ress ILC

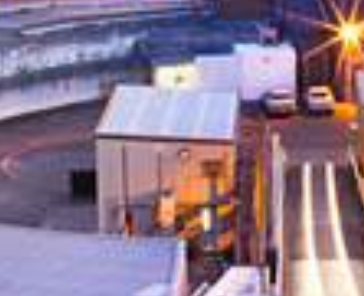

$x^{\infty}+x^{2}$



.

in 15 .

$$
\text { 过 }
$$




\section{$\underline{\text { X-Ray Diffraction }}$}



http://chemwiki.ucdavis.edu/Analytical_Chemistry/

Instrumental_Analysis/Diffraction/Powder_X-ray_Diffraction 


\section{$\underline{\text { X-Ray Diffraction Pattern }}$}

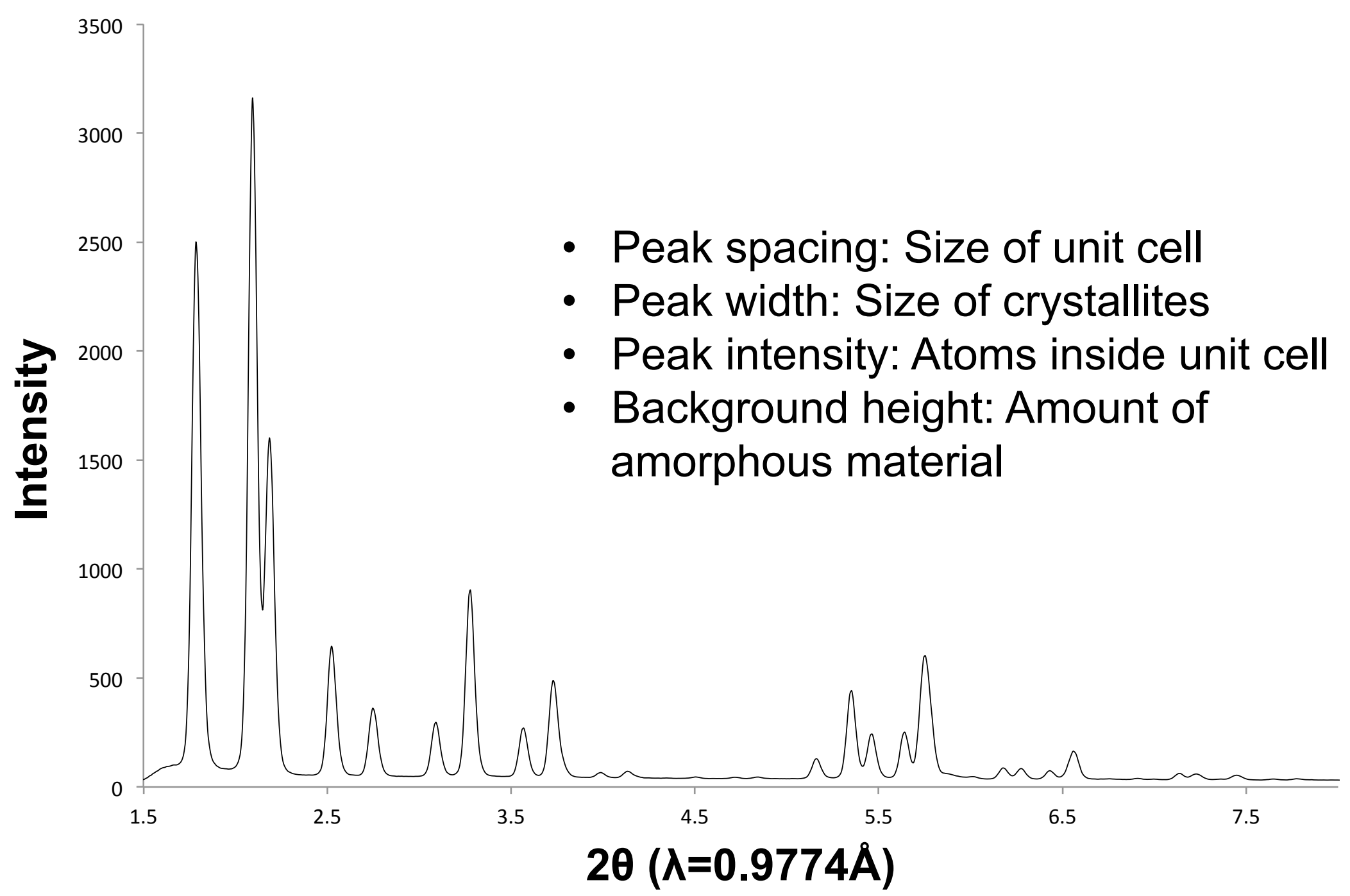




\section{MOF in Acetate Buffer Diffraction Pattern}

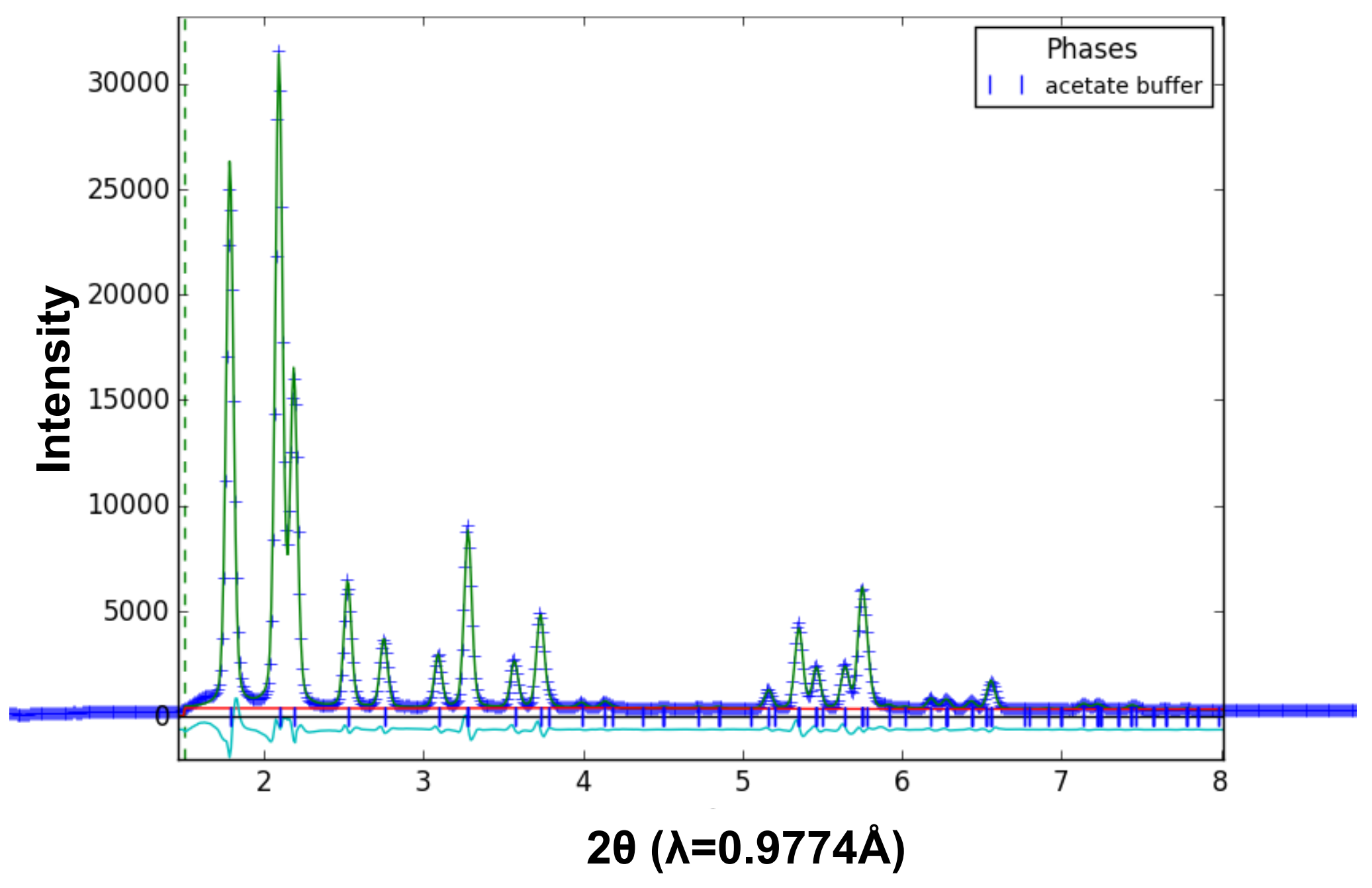




\section{Unit Cell Length of MOF for Different Samples}

\begin{tabular}{|c|c|}
\hline Sample & Unit cell length \\
\hline Only MOF & $88.0720 \pm 0.0015 \AA$ \\
\hline $\begin{array}{c}\downarrow \\
\text { MOF in Acetate } \\
\text { Buffer }\end{array}$ & $88.5120 \pm 0.0016 \AA$ \\
\hline $\begin{array}{c}\text { MOF with catalyst } \\
\text { but before catalysis }\end{array}$ & $87.19 \pm 0.04 \AA$ \\
\hline
\end{tabular}

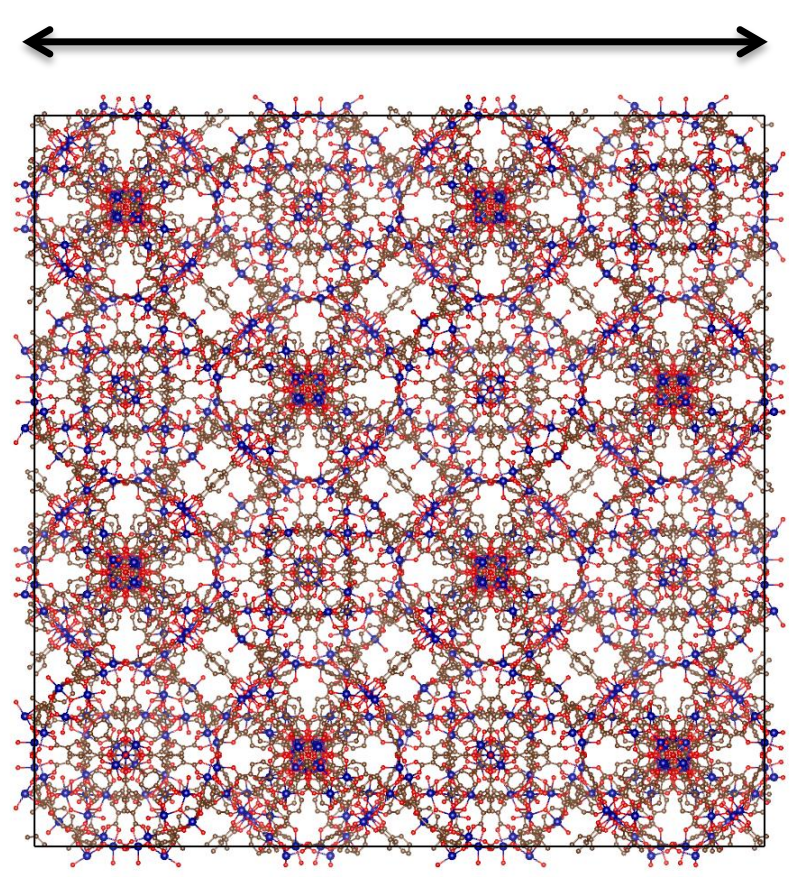

*Férey, G. et al. Science 309 (5743), 2040-2042 (2005). 


\section{Hour Catalysis Sample Overlaid with MOF in Acetate Buffer Sample}

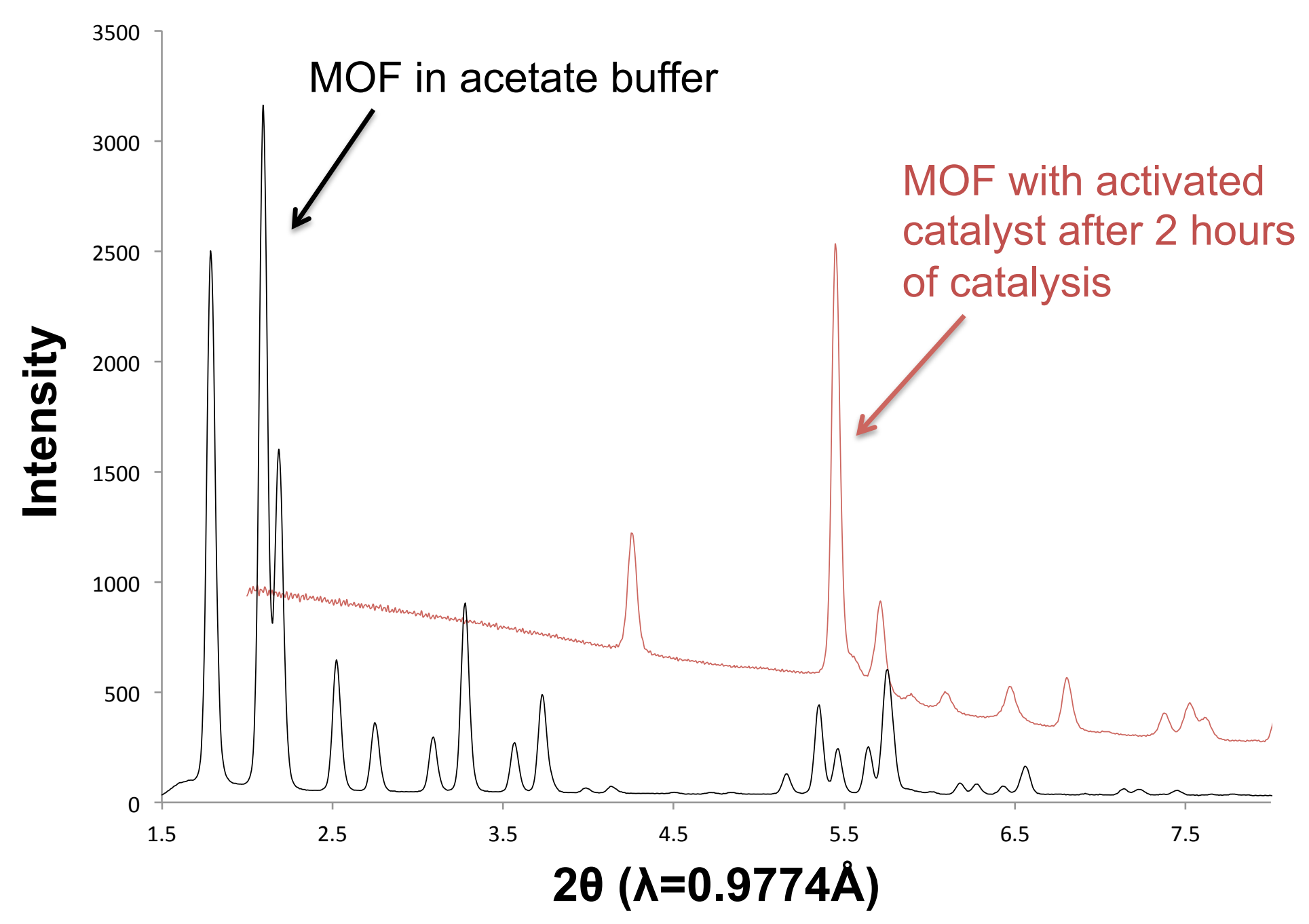




\section{Temperature Dependent Scans and In Situ Experiments}
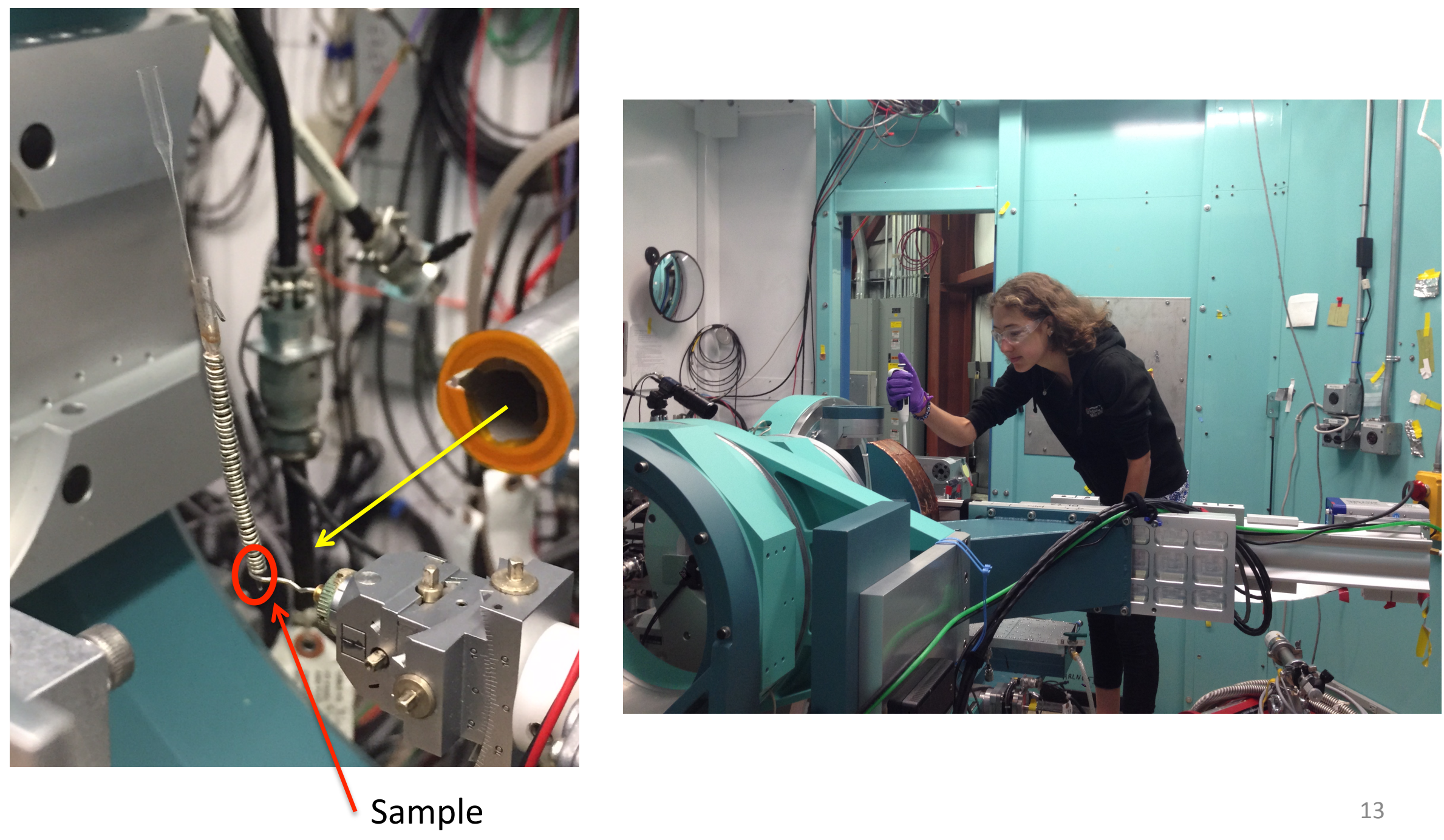


\section{Goals}

Temperature dependent scans:

- 45 minute and 2 hour catalysis samples are still good catalysts, so why?

- Split up phases by separating peaks based on heat damage (i.e. one phase might melt)

In situ experiments:

- Better understand cause of MOF degradation

- Watch MOF fall apart over course of catalysis

Results: Samples are sensitive to heat, air, and/or time 


\section{Densest Electron Density Regions in}

\section{Fourier Maps}

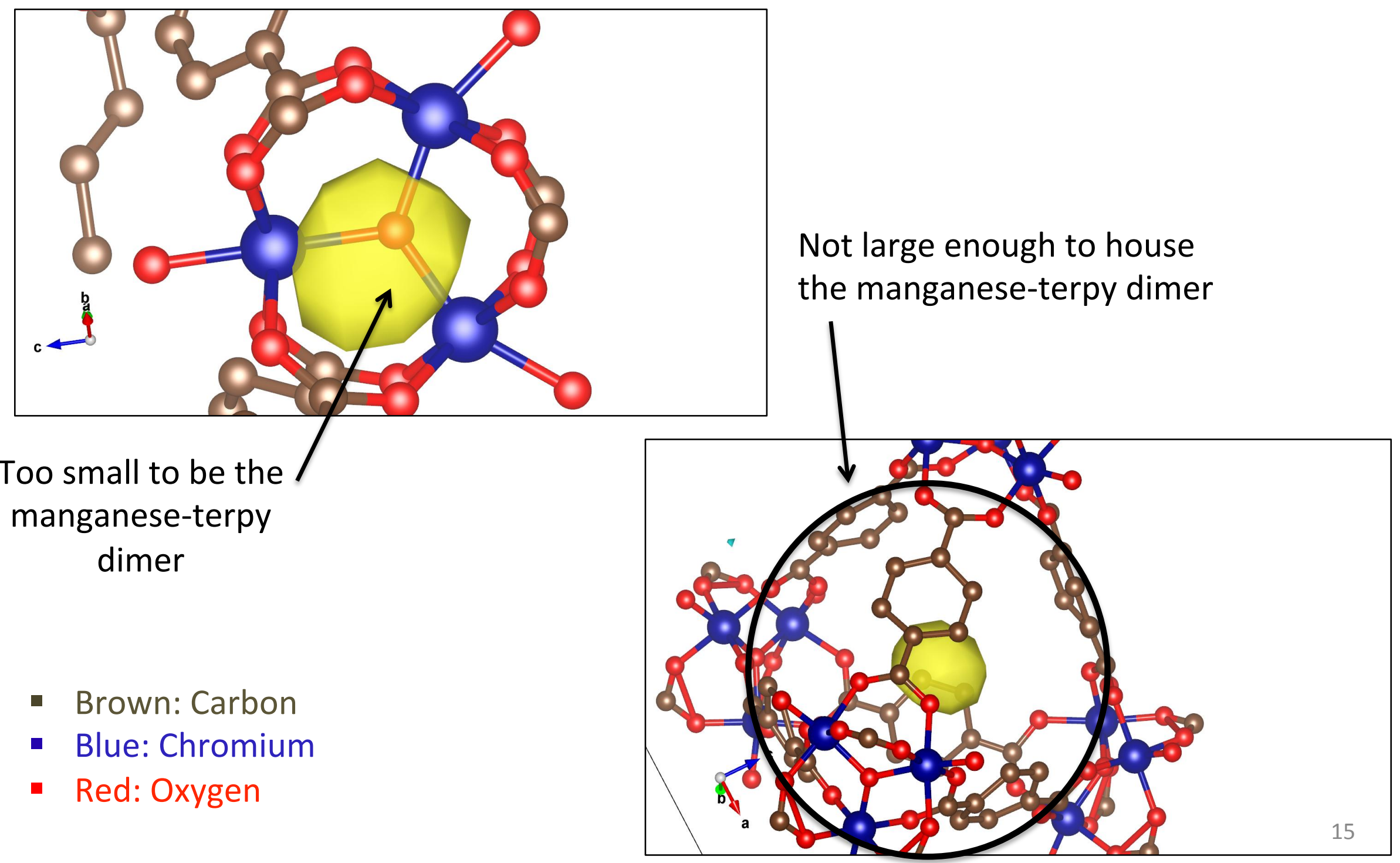




\section{Conclusions}

- There is an attractive interaction between the catalyst and MOF, which may destroy the MOF

- The manganese-terpy dimer catalyst is not the source of water oxidation catalysis in this system

- The actual catalyst might be molecular manganese oxide 


\section{$\underline{\text { Acknowledgements }}$}



Kevin Stone

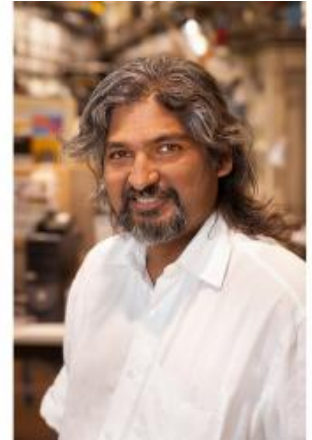

Apurva Mehta



Riti Sarangi

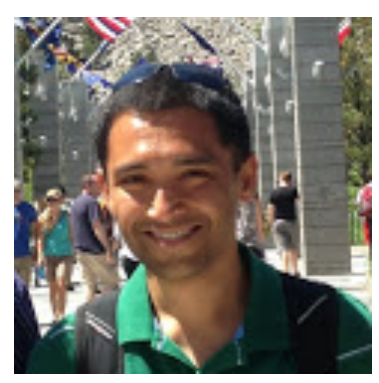

Sid Das



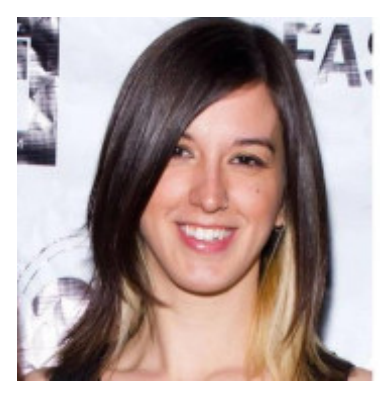

Laura Schelhas

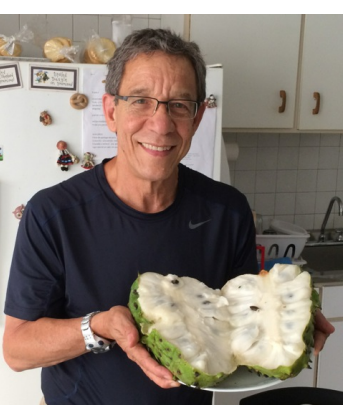

Enrique Cuellar

NATIONAL ACCELERATOR LABORATORY
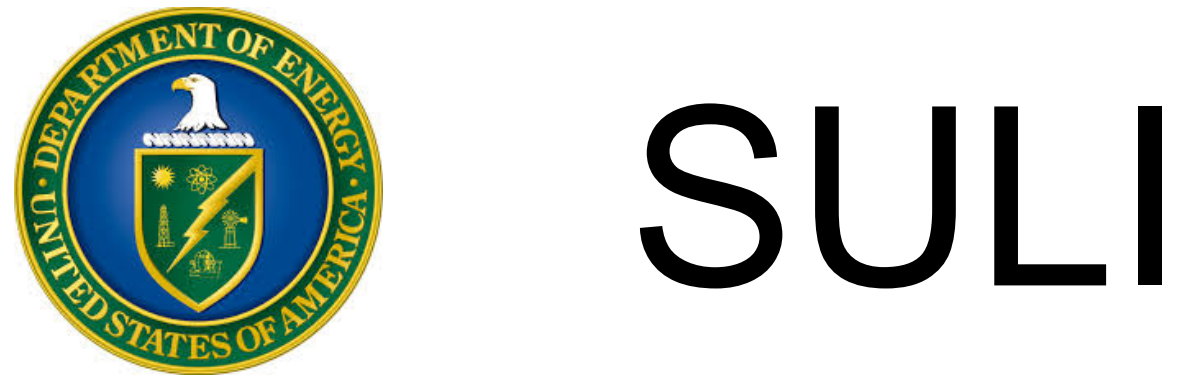


\section{$\underline{\text { Temperature Dependent Scan }}$ soo for 2 Hour Catalysis Sample}

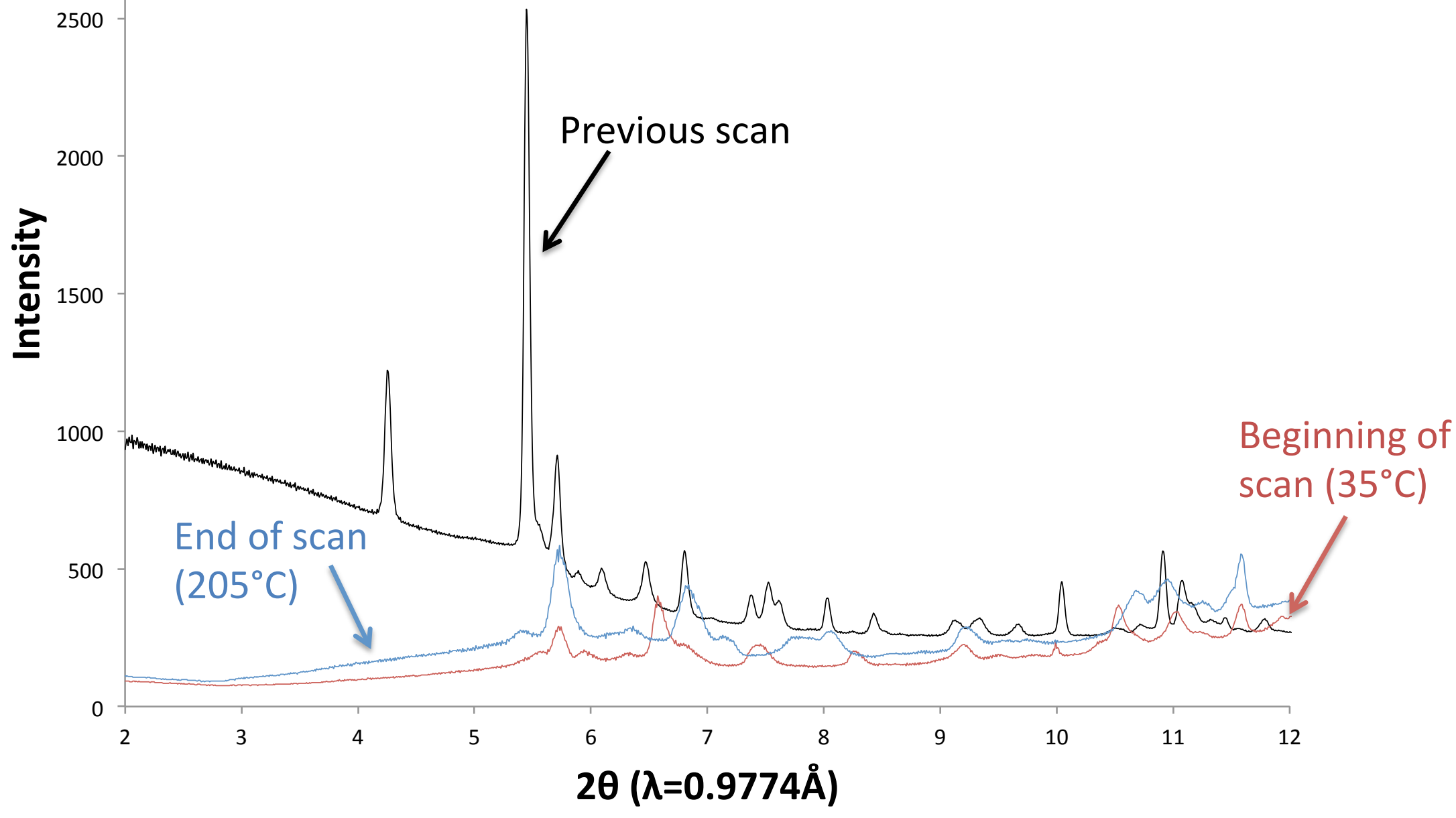

